
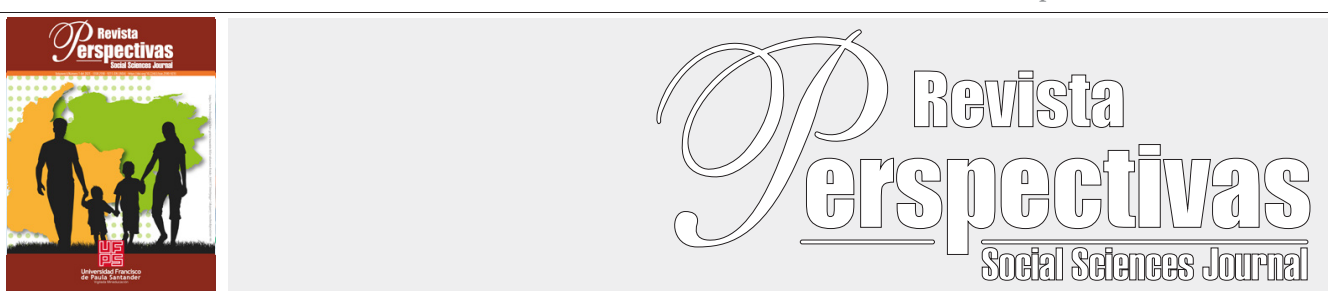

Artículo Original

https://doi.org/10.22463/25909215.2828

\title{
Relación de los sistemas propioceptivo y táctil con el desarrollo emocional en infantes
}

\section{Relationship of the propioceptive and tactile systems with the emotional development in infants}

Maria Alexandra Amaya-Mancilla'; Ludy Yanneth Peñaranda-Solano²; Yurithza Yulieth Ramirez-Rojas³, Viviana Karina Hernández-Vergel ${ }^{4}$

${ }^{1}$ Universidad de Santander UDES campus Cúcuta, Facultad de salud, grupo de investigación Entropia, Cúcuta, Colombia. 3108027058. E-mail: mar.amaya@ mail.udes.edu.co ORCID: https://orcid.org/0000-0002-8157-0288

${ }^{2}$ Universidad de Santander UDES campus Cúcuta, Facultad de salud, grupo de investigación Entropia, Cúcuta, Colombia. 3175027311.E-mail: lud.penaranda@ mail.udes.edu.co ORCID: https://orcid.org/0000-0003-0099-7675

${ }^{3}$ Universidad de Santander campus Cúcuta, Estudiante en formación de Terapia Ocupacional, Facultad de salud, del programa de Terapia Ocupacional, Cúcuta, Norte de Santander, Colombia.3224304987.E-mail: yurithza24@gmail.com ORCID: https://orcid.org/0000-0002-9979-6605

${ }^{4}$ Universidad de Santander UDES campus Cúcuta, Facultad de salud, grupo de investigación Entropia, Cúcuta, Colombia. 3188371840.E-mail: vivi.hernandez@ mail.udes.edu.co ORCID: https://orcid.org/0000-0003-3543-8419

Cómo citar: M.A Amaya-Mancilla, L.Y Peñaranda-Solano, Y.Y Ramirez-Rojas, V.R Hernández-Vergel "Relación de los sistemas propioceptivo y táctil con el desarrollo emocional en infantes ". Perspectivas, vol. 5, no. 2, pp. 30-39, 2020.

Recibido: September 12, 2019; Aprobado: November 19, 2019.

\begin{tabular}{ll}
\hline & RESUMEN \\
\hline Palabras clave: & $\begin{array}{l}\text { Esta investigación pretendía analizar de la información de los sistemas propioceptivo y táctil con el desarrollo } \\
\text { emocional de los niños y niñas de } 2 \text { a } 3 \text { años. Logrando esto por medio de la caracterización del desarrollo } \\
\text { emocional a través de la escala de valoración cualitativa del desarrollo infantil - revisada (2 años y } 1 \text { día a } 3\end{array}$ \\
Información sensorial, & $\begin{array}{l}\text { años), la determinación del sistema táctil y propioceptivo con el apoyo del perfil sensorial de Winnie Dunn } \\
\text { perfil sensorial, }\end{array}$ para así analizar la información sensorial de los niños y niñas de 2 a 3 años. Se llevó a cabo una investigación \\
sistema propioceptivo, & cuantitativa con una población de 96 menores de edad, de ambos géneros. Se obtiene una muestra de 84 \\
sistema táctil, & escolares que cursan el grado de Párvulos por medio del muestreo por conveniencia. Con la aplicación de \\
desarrollo emocional. & los instrumentos de valoración cualitativa del desarrollo infantil y el perfil sensorial de Winnie Dunn se logra \\
& identificar que el sistema táctil, es el primer sistema que tiene interacción con el ambiente y con las personas \\
& que lo rodean, por ello es el principal receptor de la parte socioafectiva como lo es el primer vínculo con la \\
madre o padre; como también el sistema propioceptivo es el encargado del posicionamiento de articulaciones y \\
músculos, es el indicador de ser brusco con sus pares, impidiendo tener una relación asertiva con sus compañeros \\
y docentes. Se concluye que los estímulos socio afectivos a temprana edad son necesarios en los niños y niñas, \\
así como también que la estimulación temprana programada potencializa el óptimo desarrollo emocional.
\end{tabular}

\section{ABSTRACT}

\section{Key words:}

Sensory information, sensory profile, proprioceptive system, tactile system, emotional development.
This research aimed to analyze information from the proprioceptive and tactile systems with the emotional development of children aged 2 to 3. This was achieved through the characterization of emotional development through the qualitative assessment scale of child development - revised ( 2 years and 1 day to 3 years), the determination of the tactile and proprioceptive system with the support of Winnie Dunn's sensory profile in order to analyze the sensory information of children from 2 to 3 years old. A quantitative research was carried out with a population of 96 children of both genders. A sample of 84 schoolchildren in the kindergarten grade was obtained by means of convenience sampling. With the application of the instruments of qualitative evaluation of the infantile development and Winnie Dunn's sensorial profile, it is possible to identify that the tactile system, is the first system that has interaction with the environment and with the people who surround it, for that reason it is the main receiver of the socio-affective part as it is the first bond with the mother or father; as also the proprioceptive system is the one in charge of the positioning of joints and muscles, it is the indicator of being abrupt with its pairs, preventing to have an assertive relation with its companions and teachers. It is concluded that socio-affective stimuli at an early age are necessary in children, as well as that early programmed stimulation potentiates optimal emotional development. 


\section{Introducción}

El Fondo de las Naciones Unidas para la Infancia, refiere que cuando ocurre el nacimiento de un ser humano, sucede una transición inmediata a un estado de indefensión respecto a la supervivencia, constituirse y desarrollar la potencialidad genética propia requiere de la intervención de personas externas que provean las herramientas necesarias para el desarrollo normal del ciclo de vida, teniendo en cuenta a su vez, que los niños en esta edad presentan una estructura psíquica inmadura en formación, que los convierte en individuos frágiles y/o vulnerables (Armus, Duhalde, Oliver, \& Woscoboinik, 2012).

Es así como los sistemas propioceptivo y táctil presentan unos receptores los cuales son los encargados de recibir el estímulo transmitiéndolo hasta el cerebro para dar respuesta. Siendo el ser humano expuesto a diversos tipos de estímulos: presión de objetos contra la piel, la adecuada posición de músculos y articulaciones, la distensión de la vejiga y la temperatura de las distintas partes del cuerpo. Cuando el estímulo puede ser dañinos ocurre una sensación somática que nos permite percibir estas circunstancias: por ejemplo, el dolor (Rosenzweig, Breedlove, Watson, \& Morgado, 2005).

Siendo la Propiocepción uno de los sistemas mediante el cual, el cerebro recoge la información sobre la posición y el movimiento de las partes del cuerpo entre sí y en relación a su base de soporte. Esto se produce a través de una serie de receptores distribuidos por todo el organismo (Miñambres, 2017). Así mismo, la toma de conciencia y la regulación en los demás y en nosotros mismos va ligado al desarrollo social-cognitivo el cual se retroalimenta entre ellos con gran fuerza, junto al desarrollo del lenguaje (Sánchez, 2020).

Desde esta perspectiva, es primordial tener en cuenta el papel que juega los sistemas propioceptivo y táctil en el desarrollo emocional de los infantes, pues, es a partir de las experiencias generadas por el medio externo y captadas a través de los receptores de dicho sistema que se logran avances significativos que apoyan la estructura de la personalidad y demás componentes emocionales que deben ser afianzados en la primera infancia.

Así mimos, se puede referir a la propiocepción como la capacidad que tiene el cuerpo humano para definir el movimiento y posición de las articulaciones. Por lo tanto, es primordial los movimientos comunes que se realizan a diario, especialmente en actividades deportivas que requieren un mayor nivel de coordinación (Ávalos \& Berrío, 2007). Por tanto, la propiocepción depende de los estímulos sensoriales: visual, auditivo, vestibular, receptores cutáneos, articulares y musculares (Diez, 2014).

Por otro lado, la temperatura se considera una influencia directa del frío o calor que abre ciertos canales iónicos, de los cuales se puede detectar tres de ellos: uno para el frío, otro para el calor, y otro para el calor extremo. Quizá haya también para el frío extremo o incluso para la templadas (Cuelliga, 2020).

De esta manera, el mundo del recién nacido se fundamenta en parámetros emocionales primarios establecidos por sus necesidades, como la relación madre e hijo y acciones básicas. Solo con el trascurrir el tiempo y a la adecuada interacción con el entorno se podrá ir dando forma al gran abanico emocional que todos tenemos (Sánchez, 2020).

Todo ello, es producido mediante los diferentes órganos de los sentidos, especialmente vista, tacto y audición, los cuales actúan como mecanismos emergentes para la adquisición de nuevo conocimiento, pues, si bien es cierto, es la mente quien se encarga de comparar y asociar experiencias sensoriales pasadas, para ser posteriormente interpretadas y conceptualizadas para abrir paso a una percepción singular. 
En concordancia con lo establecido por Velásquez (2001), el mundo externo ejerce sobre el organismo del ser humano en general y éste a su vez, reacciona a partir del estímulo percibido, sin interrumpirse el proceso de interacción. Todos los seres humanos se ven sumergidos en un entorno físico el cual ejerce determinadas acciones, siendo los sentidos en el ser humano los encargados de recibir información sobre el mundo que le rodea, teniendo en cuenta que las sensaciones se pueden considerar como base de todo conocimiento.

Las estadísticas a nivel nacional demostradas por el DANE (2016), para el año 2015 la población en edades comprendidas entre los 0 y los 4 años correspondía a 2.110.566 de habitantes de sexo femenino, logrando ocupar un $8,75 \%$ de la población total en Colombia, de igual forma, hasta la fecha existían 2.211.071 de habitantes de sexo masculino que se encontraban en el mismo rango de edad, los cuales ocupaban un $9,40 \%$ de la población total. Dichas estadísticas permiten corroborar el tamaño de la población existente a nivel nacional que requiere de atención integral para el abordaje de necesidades y potencialidades presentes a nivel físico, sensorial, social, emocional y cognitivo, para hacer frente a las necesidades del medio.

Así mismo, el Plan de Desarrollo para Norte de Santander 2016-2019 "Un Norte Productivo para todos" (Villamizar, 2016), para el 2015 del censo general del 2005, existían un total de 151.391 niños y niñas entre 0 y 5 años, los cuales pertenecen al $11,06 \%$ de la población total del territorio, la cual se encuentra entre 1.367.708; de los cuales, el 48,94\% son niñas y el 51,05\% niños. El 78,52\% habita en zona urbana, mientras que el $21,47 \%$ lo hace en zona rural.

El periodo comprendido entre los 0 y los 3 años, el ritmo y estilo de vida del ser humano se encuentra determinado por la actividad cerebral, la cual se encuentra en constante movimiento, captando los estímulos que proporciona el exterior, desde que nace, el bebé experimenta una serie de sensaciones que resultan novedosas y aumentan el nivel de alerta.

Así mismo, la primera infancia brinda una oportunidad única de colocar en marcha el desarrollo y educación de las capacidades emocionales y afectivas. El niño estará más abierto y favorable a la actividad intelectual en cuanto resuelva la necesidad de seguridad y afecto. En definitiva, la vida afectiva del niño/a es la base de la vida afectiva del adulto, de su carácter y personalidad (Mineducación, 2009).

Para el Terapeuta Ocupacional y referido en el Marco de trabajo para la práctica, cuenta con las competencias necesarias para efectuar procesos de valoración y registro de datos que puedan apoyar el proceso de praxis profesional, identificando las características del desarrollo emocional del infante en dicha etapa del ciclo vital y la caracterización del sistema propioceptivo y sistema táctil, clasificando los estímulos de acuerdo con el nivel de respuesta obtenida y los efectos en el componente emocional del menor (American Occupational Therapy Asociation, 2008).

Dezcallar (2012) estableció la relación entre los procesos mentales y sentido háptico: el cual se refiere a las emociones y recuerdos mediante el análisis empírico de texturas, donde concluye que las propiedades de las superficies que nos rodean presentan la capacidad de transmitirnos sensaciones y emociones, mostrando la importancia para la comunicación sobre estas, ya sea para evocar una sensación presente o inspirar un recuerdo posterior positivo o negativo.

\section{Según Anna Freud}

"Existe un número de circunstancias que se combinan para detener, deformar y desviar las fuerzas sobre las que se basa el crecimiento mental. No se pueden separar tajantemente los problemas o las dificultades entre sí. Todos inciden de una u otra manera como aspectos del desarrollo en los que diferentes elementos cooperan para el 
establecimiento de una problemática" (Armus, Duhalde, Oliver, \& Woscoboinik, 2012, p. 25).

En una investigación original Álvarez, Moreno \& Zea (2010) titulada "Percepciones de terapeutas ocupacionales sobre el lenguaje y la comunicación de los niños con déficit de integración sensorial", concluyeron que, al considerar, la desorganización del lenguaje es una particularidad de los niños con desordenes de integración sensorial y puede corresponder a las dificultades para secuenciar las ideas, regular su comportamiento y atender a las reglas conversacionales. Esto afecta su regulación emocional, relación con pares y adultos, su lenguaje comprensivo y expresivo.

En el año 2017 en la Fundación Universitaria Luis Amigó, Medellín, Colombia los autores Agudelo, Pulgarín \& Tabares (2017) publican un artículo llamado "La Estimulación Sensorial en el Desarrollo Cognitivo de la Primera Infancia" derivado a una investigación llamada "La Estimulación de los Sentidos en la Actividad Pedagógica con Niños y Niñas de 3 a 5 años de edad para el beneficio del Desarrollo Cognitivo en el Colegio Montessori de Medellín en el año 2016".

Permite reconocer que las experiencias sensoriales facilitan en los niños y niñas la manipulación de diferentes texturas y temperaturas donde el cuerpo, brazos y manos brindan un papel importante para el proceso de aprendizaje generando en ellos como el gusto o desagrado de la reacción ante la actividad o el material como tal, aunque muchos de los conocimientos que ellos adquieren están ligados a la exploración y la interacción de objetos de su entorno afectando positiva o negativamente la regulación de emociones y habilidades sociales (Agudelo, Pulgarín \& Tabares, 2017).

Carrascal \& Guerrero (2018) realizan investigación sobre "Implicaciones cognitiva y socio afectiva en el desarrollo del niño de 3 y 4 años, por su ingreso al preescolar a temprana edad", esta se ejecutó con el interés de determinar las implicaciones en el desarrollo de la dimensión cognitiva y dimensión socio afectiva en los niños que ingresan a temprana edad al preescolar el juego promover aprendizajes significativos a partir de procesos pedagógicos que realizan los niños por medio de juegos puedan explorar, construir, crear, inventar y por medio de estas adquieran experiencias que fortalezcan los aprendizajes.

Además, la protección integral del infante, es soportada legalmente en el marco de acción para la política de estado en el desarrollo integral de la primera infancia de cero a siempre, en tanto reconoce a los niños y a las niñas en primera infancia como sujetos de derechos, e insta al estado a la garantía y cumplimiento de los mismos, a la prevención de su amenaza o vulneración y a su restablecimiento inmediato (Arias, 2012).

\section{Materiales y métodos}

Diseño de la investigación. La investigación se plantea en un estudio de naturaleza cuantitativa, con características descriptiva, por el cual se pretende determinar el análisis y alcance de los resultados de la misma mediante una investigación de tipo descriptiva. Lerma (2016), refiere: "describir el estado, las características, factores y procedimientos presentes en fenómenos y hechos que ocurren de forma natural".

Siendo el tipo de investigación, según Lerma (2016), "constituido como un proceso sistemático, riguroso y racional de recolección, tratamiento, análisis y presentación de datos, basado en una estrategia de recolección directa de la realidad. dicha investigación es, clasificada como trabajo de campo".

Población y muestra. Para Lerma (2016), "la población es el conjunto de todos los elementos de la misma especie que presentan una característica determinada o que corresponden a una misma definición y a cuyos elementos se le estudiaran las características y relaciones". Por tal razón, se puede 
afirmar que la población objeto de estudio está constituida por 96 escolares aproximadamente de la Educación Escolar de prescolar, con edades que oscilan entre los 2 a 3 años de ambos sexos.

Así mismo, la muestra se considera como no probabilística, según los (criterio de inclusión), menores de 2 a 3 años, se incluyó los menores con asentimiento informado por padres de familia para participar en la investigación.

De acuerdo con lo anterior se evidencia una muestra de siendo 84 escolares en el rango de edades de 2 a 3 años encontrándose en el grado de párvulos.

Para la presente investigación, se tiene presente las consideraciones éticas, donde se cuenta con las siguientes recomendaciones para la investigación en seres humanos. En términos prácticos se efectúa con los tres principios éticos comúnmente aceptados en investigaciones biomédica, el cual se determinan a continuación:

- Principio de respeto a la persona o a la autonomía: cada participante podrá decidir de forma voluntaria e informada la participación en la investigación.

- Principio de no maleficencia: la prioridad en la investigación es no perjudicar a ninguno de los participantes del estudio.

- Principio de beneficencia: esta investigación tiene como fin, maximizar el beneficio para los participantes del estudio.

Uso de asentimiento informado. cada uno de los autores debe presentar en detalle los beneficios, riesgos y consideraciones del proceso en un lenguaje sencillo para que los participantes puedan ejercer su libre albedrío, de igual forma se conserva la decisión de interrumpir la participación en cualquier momento. Esto a fin de solicitar la autorización a los participantes del estudio para que suministren información y esta pueda ser utilizado en la investigación. Las consideraciones a tener presentes:
- Comunicación a la persona sobre los procesos que se van a realizar, riesgos e incomodidades, así como sus derechos.

- Declaración de que la participación es voluntaria y que la negativa a participar o a retirarse en cualquier momento no implica represalias, castigos o pérdida de algún beneficio.

- Compromiso del investigador responsable sobre la confidencialidad de los datos (Fundación investigación biomédica, 2011).

Instrumentos de recolección de información. La técnica que se utilizó para el desarrollo y aplicabilidad de los instrumentos fue la observación, la cual es un "procedimiento de recolección de datos e información que consiste en utilizar los sentidos para observar hechos y realidades sociales presentes y a la gente donde desarrolla normalmente sus actividades" (Soledad, s.f., p.3).

El instrumento que se utilizó para realizar la recolección de datos fue "escala de valoración cualitativa del desarrollo infantil desde el nacimiento hasta los seis años" del Instituto Colombiano de Bienestar Familiar, el cual solo se va a tomar el rango de edad 2 años y un día hasta los 3 años; la segunda es "el perfil sensorial de Winni Dunw" quien está dirigida desde los 36 meses hasta los 11 años; la cual brindo la comparación de los sistemas propioceptivo y táctil con el desarrollo emocional.

\section{Resultados}

De acuerdo al proceso de evaluación del desarrollo infantil por medio de la interacción de los infantes con sus pares, personas y entorno, se le aplican a niños y niñas de 0 a 6 años, de acuerdo con el proyecto se tomó el rango de edad de 2 años y un día a 3 años. El instrumento valora las actividades espontaneas y naturales de los niños y niñas al interactuar con sus pares, padres, cuidadores, familia, docentes; seguidamente caracterizo la relación con los demás, relación consigo mismo y relación con el mundo. Este instrumento se aplicó a 84 niños en edades comprendidas entre los 2 a 3 años. 
Tabla I. Distribución porcentual según relación con los demás de la escala valoración cuantitativa del desarrollo infantil, aplicada a los niños y niñas de 2 a 3 años

\begin{tabular}{|c|c|c|c|c|c|c|c|c|c|c|}
\hline \multirow{4}{*}{ CRITERIO } & \multicolumn{10}{|c|}{ RELACIÓN CON LOS DEMAS } \\
\hline & \multicolumn{4}{|c|}{ COMUNICACIÓN } & \multicolumn{4}{|c|}{ INTERACCIÓN } & \multirow{2}{*}{\multicolumn{2}{|c|}{$\begin{array}{c}\text { CONSTRUCCIÓN } \\
\text { DE NORMAS }\end{array}$}} \\
\hline & \multicolumn{2}{|c|}{ VERBAL } & \multicolumn{2}{|c|}{ NO VERBAL } & \multicolumn{2}{|c|}{ INDEPENDENCIA } & \multicolumn{2}{|c|}{ COOPERACIÓN } & & \\
\hline & $\mathrm{N}^{\circ}$ Esc & $\%$ & $\mathrm{~N}^{\circ}$ Esc & $\%$ & $\mathrm{~N}^{\circ} \mathrm{Esc}$ & $\%$ & $\mathrm{~N}^{\circ}$ Esc & $\%$ & $\mathrm{~N}^{\circ}$ Esc & $\%$ \\
\hline Avanzado & 18 & $21 \%$ & 20 & $24 \%$ & 47 & $56 \%$ & 0 & $0 \%$ & 29 & $35 \%$ \\
\hline Esperado & 32 & $38 \%$ & 38 & $45 \%$ & 30 & $36 \%$ & 78 & $93 \%$ & 27 & $32 \%$ \\
\hline En riesgo & 34 & $40 \%$ & 26 & $31 \%$ & 7 & $8 \%$ & 6 & $7 \%$ & 28 & $33 \%$ \\
\hline Total & 84 & $100 \%$ & 84 & $100 \%$ & 84 & $100 \%$ & 84 & $100 \%$ & 84 & $100 \%$ \\
\hline
\end{tabular}

De acuerdo con la Tabla I se puede establecer que el $40 \%$ correspondiente a 34 escolares presentan riesgo en la comunicación verbal, debido a que no refieren una frase de tres o más palabras para expresar lo que desea o no, mostrando falencias en la socialización con sus pares o cuidadores; de igual manera un $31 \%$ de 26 escolares se encuentran a riesgo en la comunicación no verbal, interfiriendo en la expresión de los sentimientos o emociones corporales, sin embargo logran identificar representaciones de situaciones de la vida diaria; por otro lado el 33\% implica 28 infantes presentando riesgo en la autonomía debido al no poder controlar los esfínteres, no manifestando incomodidad cuando tiene el pañal mojado, de igual forma no logran seguir instrucciones sencillas de dos pasos; aunque presenta $93 \%$ correspondiente a 78 escolares se encuentran esperando a cooperar con sus pares o cuidadores.

Tabla II. Distribución porcentual según relación consigo mismo de la escala valoración cuantitativa del desarrollo infantil, aplicada a los niños y niñas de 2 a 3 años

\begin{tabular}{|c|c|c|c|c|c|c|c|c|}
\hline \multirow{4}{*}{ CRITERIO } & \multicolumn{8}{|c|}{ RELACIÓN CON LOS CONSIGO MISMO } \\
\hline & \multicolumn{4}{|c|}{ IDENTIDAD } & \multirow{2}{*}{\multicolumn{2}{|c|}{ AUTOESTIMA }} & \multirow{2}{*}{\multicolumn{2}{|c|}{$\begin{array}{l}\text { MANEJO } \\
\text { CORPORAL }\end{array}$}} \\
\hline & \multicolumn{2}{|c|}{$\begin{array}{l}\text { PERSONAL Y } \\
\text { DE GENERO }\end{array}$} & \multicolumn{2}{|c|}{ SOCIAL } & & & & \\
\hline & $\mathrm{N}^{\circ}$ Esc & $\%$ & $\mathrm{~N}^{\circ}$ Esc & $\%$ & $\mathrm{~N}^{\circ}$ Esc & $\%$ & $\mathrm{~N}^{\circ}$ Esc & $\%$ \\
\hline Avanzado & 56 & $67 \%$ & 0 & $0 \%$ & 29 & $35 \%$ & 78 & $93 \%$ \\
\hline Esperado & 0 & $0 \%$ & 32 & $38 \%$ & 25 & $30 \%$ & 5 & $6 \%$ \\
\hline En riesgo & 28 & $33 \%$ & 51 & $61 \%$ & 28 & $33 \%$ & 1 & $1 \%$ \\
\hline Total & 84 & $100 \%$ & 84 & $100 \%$ & 84 & $100 \%$ & 84 & $100 \%$ \\
\hline
\end{tabular}

De acuerdo con la Tabla II se puede identificar que un $61 \%$ correspondiente a 51 escolares se encuentran a riesgo a nivel de identidad social presentando falencias en al no identificar ni nombrar las emociones básicas en sí mismo; el 33\% de 28 escolares presentan riesgo en cuanto al autoestima debido a las falencias de no mostrar a los demás lo que han hecho, con gusto y orgullo, también en no expresar verbalmente o gestualmente lo que le gusta o disgusta, no requiere que le reconforten de manera permanente, tampoco hace preguntas en repetidas ocasiones. Sin embargo, en el manejo corporal se encuentran avanzado en cuanto al rango de edad teniendo en cuenta el desarrollo motor como lo es la coordinación gruesa y fina.

Tabla III. Distribución porcentual según relación con el mundo de la escala valoración cuantitativa del desarrollo infantil, aplicada a los niños y niñas de 2 a 3 años

\begin{tabular}{lcccccc}
\hline \multirow{3}{*}{ CRITERIO } & \multicolumn{5}{c}{ RELACIÓN CON LOS CONSIGO MISMO } \\
\cline { 2 - 8 } & \multicolumn{2}{c}{ IDENTIDAD } & AUTOESTIMA & MANEJO CORPORAL \\
\cline { 2 - 7 } & $\mathrm{N}^{\circ}$ Esc & $\%$ & $\mathrm{~N}^{\circ}$ Esc & $\%$ & $\mathrm{~N}^{\circ}$ Esc & $\%$ \\
\hline Avanzado & 29 & $35 \%$ & 31 & $37 \%$ & 37 & $44 \%$ \\
\hline Esperado & 3 & $4 \%$ & 52 & $62 \%$ & 0 & $0 \%$ \\
\hline En riesgo & 52 & $62 \%$ & 1 & $1 \%$ & 47 & $56 \%$ \\
\hline Total & 84 & $100 \%$ & 84 & $100 \%$ & 84 & $100 \%$ \\
\hline
\end{tabular}

De acuerdo con la Tabla III se evidencia que el $62 \%$ correspondiente a 52 escolares se encuentra a riesgo en el reconocimiento de objetos como lo es relacionarlos con los usos comunes de cada uno, interfiriendo en la proporción de tamaño y ubicación espacial; por otro lado en representación de la realidad social se encuentran en riesgo con un 58\% debido a que no logran relacionar vestuarios u objetos, con los diferentes ocupaciones o roles de las personas; sin embargo, en las relaciones de causalidad tienen un $62 \%$ donde están a nivel normal del desarrollo cronológico.

De acuerdo con los resultados derivados a través de la recopilación de datos del instrumento perfil sensorial de Winnie Dunn, teniendo en cuenta los criterios de aguante y tono (sistema propioceptivo) y procesamiento táctil (sistema táctil), el cual lo tenían que responder los cuidadores o docentes de los escolares, viendo la interacción dentro y fuera del aula de clase el desarrollo. Este instrumento se aplicó a 84 niños en edades comprendidas entre los 2 a 3 años. 
Tabla IV. Distribución porcentual según perfil sensorial, del sistema táctil y propioceptivo, aplicada a los niños y niñas de 2 a 3 años.

\begin{tabular}{|c|c|c|c|c|c|c|}
\hline \multicolumn{7}{|c|}{ PERFIL SENSORIAL DE LOS ESCOLARES DE $2 \mathrm{~A} 3$ AÑOS } \\
\hline \multirow[t]{2}{*}{ PPROCESAMIENTO } & \multicolumn{2}{|c|}{$\begin{array}{c}\text { DIFERENCIA } \\
\text { PROBABLE }\end{array}$} & \multicolumn{2}{|c|}{ FUNCION TIPICA } & \multicolumn{2}{|c|}{$\begin{array}{l}\text { DIFERENCIA } \\
\text { DEFINITIVA }\end{array}$} \\
\hline & $\begin{array}{l}\text { NUMERO DE } \\
\text { ESCOLARES }\end{array}$ & $\%$ & $\begin{array}{l}\text { NUMERO DE } \\
\text { ESCOLARES }\end{array}$ & $\%$ & $\begin{array}{l}\text { NUMERODE } \\
\text { ESCOLARES }\end{array}$ & $\%$ \\
\hline Procesamiento táctil & 14 & $17 \%$ & 30 & $36 \%$ & 40 & $48 \%$ \\
\hline Procesamiento propioceptivo & 10 & $12 \%$ & 33 & $39 \%$ & 41 & $49 \%$ \\
\hline
\end{tabular}

De acuerdo con la Tabla IV se muestra que el $48 \%$ de los escolares presentan una diferencia definitiva en el sistema de procesamiento táctil, debido a las falencias en el procesamiento evitando el contacto con el entorno o ambiente que lo rodea, donde no toleran que lo abracen; en cuanto al sistema propioceptivo se encuentra a riesgo un $49 \%$ de los escolares mostrando falencias en la modulación sensorial en cuanto aguante y tono.

\section{Conclusión y discusión}

Inicialmente se aplicó la escala de valoración cualitativa del desarrollo infantil revisada de (2 años y 1 día a 3 años) de la política de 0 a siempre Fundación Saldarriaga Concha validado por la Pontificia Universidad Javeriana, dicho instrumento analiza tres grandes ítems (1. Desarrollo con los demás; 2. Desarrollo consigo mismo; 3. Desarrollo con el mundo) teniendo en cuenta los rangos de edad 2 años y un día hasta los 3 años, permitiendo caracterizar el desarrollo emocional de los niños; seguidamente se utilizó el según instrumento que fue el Perfil sensorial de Winni Dunw el cual estudia (1. procesamiento táctil (sistema táctil); 2. el procesamiento de aguante y tono (sistema propioceptivo); 3. Reacciones emocionales y de comportamiento), logrando determinar el sistema táctil y propioceptivo, aplicando solo estos dos criterios de la prueba con ayuda del $70 \%$ a los docentes y el $30 \%$ a los padres.

La presente investigación se llevó a cabo con una población constituida por 147 escolares aproximadamente de la Educación Escolar de prescolar, con edades que oscilan entre los rangos de edad de 2 a 3 años donde abarca una población de 96 infantes. Estos niveles se seleccionaron considerando lo consultado en los antecedentes la mayor frecuencia de casos de acoso escolar se registra durante el Tercer Ciclo lo cual tiende a disminuir en la Educación Media.

Dentro de la investigación se formuló como pregunta ¿Cuál es la asociación de la información de los sistemas propioceptivo y táctil con el desarrollo emocional de los niños y niñas de 2 a 3 años de Cúcuta norte de Santander? dando respuesta con los resultados obtenidos donde se observa que las falencias en el procesamiento, hace que los niños eviten el contacto con el entorno o ambiente que lo rodea, donde no toleran que los abracen por consiguiente son infantes que tienen respuestas de tipo emocional (negativas) observamos que si el infante no logra nombrar o identificar emociones básicas como tipo respuesta ante algún estimulo se ve afectado su desempeño generando respuestas negativas como "llanto o lucha" al momento del corte de cabello, uñas o de ir al dentista, debido que dichos estímulos sensorialmente son molestos para él, lo anterior denota que se hace pertinente programar la estimulación temprana en la primera infancia para potencializar el oprimo desarrollo emocional en los niños, logrando comprobar la segunda hipótesis planteada en la presente investigación.

En cuanto a los resultados obtenidos frente a los antecedentes presentados, se logra analizar que algunos estudios realizados en el año 2012 por la autora Teresa Dezcallar Saéz sobre la "Relación entre los procesos mentales y sentido háptico: emociones y recuerdos mediante el análisis empírico de texturas" la cual pretendía examinar la vinculación entre la sensibilidad perceptiva háptica y su correlación con los estados subjetivos, como son la parte emocional y de recuerdos suscitados por vía táctil.

La investigación se direcciona a explorar directamente a los vínculos posibles entre las texturas y los recuerdos emocionales; teniendo como referente los tres tipos de percepción: percepción 
táctil la que hace referencia a la información que se obtiene solo a través de la piel y es producida por un determinado estimulo.

Por consiguiente, el anterior estudio se relaciona con los resultados adquiridos en nuestra investigación ya que los resultados de esta refiere la relación entre las texturas, las emociones y los recuerdos $\mathrm{y}$ en nuestra investigación vemos que los infantes que muestran rechazo a estímulos táctiles o propioceptivos responden negativamente mostrando (Odio, tristeza, llanto y aversión) ante diferentes situaciones tales como (Ser tocados, lavado de dientes, ir al dentista, cortado de cabello, o se cuelgan de las cosas, evitan ensuciarse, aprietan sus articulaciones o se vuelven rígidas con dichos comportamientos, sus músculos son débiles y esto afectara su desempeño ocupacional funcional (En su aprendizaje, relaciones interpersonales) puesto que son escolares que no logran terminar actividades, no siguen instrucciones, hacen pataletas constantes al ser expuestos al estímulo.

Por lo tanto, las dos investigaciones tienen relación en sus resultados puesto que se confirma que el desarrollo emocional se asocia con el sentido táctil y propioceptivo.

Los resultados presentados se tamizan a la luz de marco teórico que sustentan la investigación, argumentando que el ambiente humano del infante debe estar caracterizado por el suministro de sensaciones positivas, las cuales desencadenen respuestas adaptativas exitosas que benefician el nivel de respuesta emocional, dentro de las acciones más representativas para el ser humano en la etapa de primera infancia se encuentran las expresiones de afectos como lo son los besos, caricias y diversos signos de unión con él, cuyas sensaciones son percibidas por medio del input de los órganos sensoriales que trabajan en conjunto con el sistema propioceptivo y táctil para traducir la información y en dicho caso, generar una respuesta placentera que contribuya en el estrechamiento de los lazos de unión con él. Se evidencia que los niños y niñas que presentan falencias en el sistema sensorial táctil y propioceptivo le impiden la modulación adecuada de la información proveniente del medio lo que ocasiona que exageren sus movimientos, rechacen los estímulos y se tornen inestables emocionalmente.

La investigación realizada por Velásquez (2001), "el mundo exterior actúa sobre el organismo en general y éste reacciona a partir de lo percibido, sin interrumpirse ningún momento este proceso de interacción". Todos los seres humanos se ven inmersos en un contexto físico el cual ejerce determinadas acciones.

Es decir que cada estimulo sensorial de tipo táctil o propioceptivo va a generar una respuesta adaptativa generando una determinada acción, sin embargo, aquellos infantes que presentan dificultades sensoriales de tipo táctil y propioceptivo generan respuestas negativas por medio de su comportamiento afectando el desarrollo emocional del infante.

Se puede concluir que los menores de 2 a 3 años de edades mostraron falencias en la socialización con sus pares o cuidadores interfiriendo en la expresión de los sentimientos o emociones corporales, no identifican ni nombran emociones básicas en sí mismos, 28 escolares presentan riesgo en cuanto al autoestima debido a las falencias de no mostrar a los demás lo que han hecho, con gusto y orgullo, tampoco expresan verbalmente o gestualmente lo que le gusta por lo tanto su reacción es negativa ante la exposición de dichos estímulos que rechazan como "usar texturas que no les agraden" "ser tocados" "ser expuestos ante posturas incomodas debido a que sus músculos son débiles o sus articulaciones son rígidas".

A nivel global se encontró riesgo en el reconocimiento de objetos como lo es relacionarlos con los usos comunes de cada uno, interfiriendo en la proporción de tamaño y ubicación espacial; por 
otro lado, en representación de la realidad social se encuentran en riesgo debido a que no logran relacionar vestuarios u objetos, con los diferentes ocupaciones o roles de las personas.

Se observó que tras la aplicación del perfil sensorial que los infantes presentan una diferencia definitiva en el sistema de procesamiento táctil, debido a las falencias en el procesamiento evitando el contacto con el entorno o ambiente que lo rodea, donde no toleran que lo abracen; en cuanto al sistema propioceptivo se encuentra a riesgo mostrando falencias en la modulación sensorial en cuanto aguante y tono debido a que aprietan débilmente, sus movimientos son rígidos, se cansan fácilmente ante una postura específica, siempre se apoyan; repercutiendo en la ejecución de actividades, seguimiento de instrucciones, mantenerse en el puesto y finalizar una actividad.

De igual manera se puede concluir que el sistema táctil, siendo el primer sistema que tiene interacción con el ambiente y con las personas que lo rodean, por ello es el principal receptor de la parte socioafectiva como lo es el primer vínculo con la madre o padre, como también el sistema propioceptivo que es el encargado del posicionamiento de articulaciones y músculos, es el indicador de ser brusco con sus pares, impidiendo tener una relación asertiva con sus compañeros y docentes dentro de la institución educativa.

\section{Referencias}

Agudelo, L., Pulgarín, L., \& Tobares, C. (2017). La estimulación Sensorial en el desarrollo cognitivo de la primera infancia. Revista Fuentes, 19(1), 7383. Recuperado de http://dx.doi.org/10.12795/ revistafuentes. 2017.19.1.04

Álvarez, B., Moreno, M., \& Zea, P. (2010). Percepciones de terapeutas ocupacionales sobre el lenguaje y la comunicación de los niños con déficit de integración sensorial. Revista Facultad de Medicina, Universidad Nacional de Colombia,
58(4), 263-271.

American Occupational Therapy Asociation (2008). Occupational therapy practice framework: Domain and process. American Journal of Occupational Therapy, 62, 625-683.

Arias, F. (2012). El proyecto de investigación (6a ed.). Caracas: Episteme.

Armus M., Duhalde C., Oliver M., \& Woscoboinik N. (2012). Desarrollo emocional. Clave para la primera infancia de 0 a 3 años (1a ed.). Argentina: UNICEF.

Asociación Probienestar de la Familia Colombiana - Profamilia. (2011). Encuesta Nacional de Demografía y Salud (ENDS 2010). Bogotá: Printex Impresores Ltda. Recuperado de https:// profamilia.org.co/wp-content/uploads/2018/12/ ENDS-2010.pdf

Ávalos, C. N., \& Berrío J. A. (2007). Evidencia del trabajo propioceptivo utilizado en la prevención de lesiones deportivas (trabajo especialista). Universidad de Antioquia, Medellín, Colombia.

Carrascal, Y., \& Guerrero, J. (2018). Implicaciones cognitiva y socio afectica en el desarrollo del niño de 3 y 4 años, por su ingreso al preescolar a temprana edad (tesis pregrado). Universidad de Francisco de Paula Santander, Ocaña, Norte de Santander.

Cuelliga, M. (2020). El sentido del tacto y la influencia en el ser. Nuue, Revista de promoción de la investigación. Recuperado de https:// www.revistanuve.com/el-sentido-del-tacto-y-lainfluencia-en-el-ser/

DANE (2016). Pirámide de población total según sexo y grupos quinquenales de edad. Recuperado de https:/geoportal.dane.gov.co/midaneapp/pob. html. 
Dezcallar, T. (2012). Relación entre procesos mentales y sentidos háptico: emociones $\mathrm{y}$ recuerdos mediante el análisis empírico de texturas (tesis doctoral). Universidad Autónoma de Barcelona, Barcelona, España.

Diez, E. (2014). La propiocepción como método de prevención de lesiones (trabajo de Grado). Universidad del León, Venganza, Chile.

Fundación investigación biomédica. (2011). Aspectos éticos en la investigación biomédica. Recuperado de http://www.iisgm.com/wpcontent/uploads/2011/09/Aspectos-Eticos-enInvestigaci\%C3\%B3n-Biom\%C3\%A9dica.pdf

Galbán, P., Sansó, F., Díaz-Canel, A., Carrasco, M., \& Oliva, T. (2007). Envejecimiento poblacional y fragilidad en el adulto mayor. Rev Cubana Salud Pública, 33(1), 1-17. Recuperado de http://scielo.sld.cu/scielo.php?script=sci arttext\&pid=S0864-34662007000100010

Lerma, H. (2016). Metodología de la investigación: Propuesta, anteproyecto y proyecto (5th ed.). Bogotá: Eco Ediciones.

Miñambres, D. (2017). ¿Qué es el Sistema Propioceptivo en Fisioterapia y el deporte? Recuperado de https:// rehabilitacionpremiummadrid.com/blog/diegominambres/que-es-el-sistema-propioceptivopropiocepcion-en-fisioterapia-y-deporte/

Ministerio de Educación Nacional (Mineducación). (2009). Desarrollo infantil y competencias en la primera infancia. Bogotá, Colombia: Mineducación. Recuperado de https://www. mineducacion.gov.co/primerainfancia/1739/ articles-178053_archivo_PDF_libro_ desarrolloinfantil.pdf

Rosenzweig M, Breedlove M, Watson N. \& Morgado, I. (2005). Psicobiología: una introducción a la neurociencia conductual, cognitiva y clínica. Barcelona: Ariel.

Sánchez, E. (2020). Desarrollo Emocional en la infancia. Recuperado de https://www.psicoactiva. com/blog/desarrollo-emocional-en-la-infancia/

Soledad, M. (s.f.). Las técnicas de investigación: La observación . Recuperado de https://www. academia.edu/36157300/Las_t $\%$ C3\%A9cnicas de_investigaci $\%$ C $3 \%$ B $3 n_{-} 1 a_{-}$ observaci\%C3\%B3n

Velásquez, J. (2001). Curso Elemental de Psicología. México: Selector

Villamizar, W. (2016). Plan de desarrollo Norte de Santander 2016-2019. Un Norte productivo para todos. Recuperado de http://www. sednortedesantander.gov.co/sitio/images/ documentos/informesdelsector/PDD $\% 20$ NDS\%202016-2019.pdf 